\title{
Seeing the Complete Picture: Imaging in Prostate Cancer
}

\author{
Layla Southcombe \\ Editorial Assistant
}

Citation: EMJ Urol. 2020;8(Suppl 2):19-21.

\begin{abstract}
UIDELINES by the European Association of Urology (EAU) for managing prostate cancer (PCa) were updated at last year's EAU meeting, and have had profound implications on the PCa diagnostic pathway. One crucial set of changes in the recommendations was that every time a biopsy is considered necessary based on risks, an MRI needs to be conducted prior to the biopsy. Given this paradigm shift towards imageguided diagnostics, the future landscape of imaging in PCa looks to be one containing innovative approaches.
\end{abstract}

\section{MRI}

\section{ULTRASOUND}

Overdiagnosis and overtreatment are two crucial criticisms in $\mathrm{PCa}$ that MRI could help diminish. In the Plenary session "Modern prostate cancer imaging in daily practice," Prof Boris Hadaschik, University Hospital Essen, Essen, Germany, outlined the use of MRI in PCa, highlighting that "MRI is an excellent tool to help us in finding the most relevant cancer in the prostate because we not only can look at anatomy, but also cell density and perfusion," both key hallmarks of cancerous tumours.

Prof Hadaschik highlighted a comparative study of MRI versus transrectal ultrasound scan for the detection of clinically significant $\mathrm{PCa}$ in which MRI was far superior to transrectal ultrasound scan, having a sensitivity of $93 \%$ versus $48 \%$, respectively.' While MRI is not the perfect method for diagnosing and grading PCa, it does allow for the detection of the bigger and more aggressive lesions, and the integration of MRI has been shown to improve PCa risk modelling. ${ }^{2}$
Historically, the reliability of ultrasound for detecting PCa has come under question and diagnostic performances have not been superior to systematic biopsy, despite the number of different modalities developed. The cost and availability of MRI are two key concerns of the MRI pathway and therefore ultrasound, as an inexpensive and convenient tool, may have a place in PCa diagnosis, but only if the accuracy of the modalities can be improved.

In juxtaposition to the qualitative interpretation of contrast-enhanced ultrasound, contrastultrasound dispersion imaging (CUDI) makes use of time intensity curves to analyse contrast dispersion dynamics. In another presentation in the Plenary session, Dr Christophe Mannaerts, Amsterdam University Medical Center, Amsterdam, the Netherlands, recounted data from a recent study investigating detection rates of CUDI-targeted biopsy, MRI-targeted biopsy, and systematic biopsy. ${ }^{3}$ Despite both CUDI- and 
MRI-targeted biopsy reducing the detection of Grade Group 1 PCa, both approaches missed substantial amounts of Group 2 or higher PCa when compared with systematic biopsy. In comparison to MRI, CUDI had comparable negative predictive value, but a lower positive predictive value, with more false positives most likely due to benign prostatic hyperplasia or prostatitis. While these results question the sole use of CUDI, Dr Mannaerts posed the idea of a multiparametric approach to ultrasound having value.

\section{Multiparametric Ultrasound}

PCa is a notoriously multifocal and heterogenous disease, and therefore taking a multiparametric ultrasound approach that combines complementary biomarkers, including tissue texture, elasticity, and perfusion, could facilitate accurate detection of clinically significant PCa, noted Dr Mannaerts. Analysis of a multiparametric ultrasound combination of grey-scale, contrastenhanced, sheer wave elastography, and CUDI ultrasound showed a significant improvement in sensitivity (ranging from $74 \%$ for Epstein criteria to $85 \%$ for International Society of Urological Pathology [ISUP] Grade 3 or higher). ${ }^{4}$ Overall, the multiparametric approach outperformed the modalities as singulars on a pixel-wise and region-wise analysis. Interestingly, the potential for the integration of machine learning into the approach was technically feasible and demonstrated in the study. Dr Mannaerts concluded that these findings support the rationale for further defining the usefulness of multiparametric ultrasound in the diagnostic pathway of PCa.

\section{PSMA PET/CT}

Prostate specific membrane antigen (PSMA) PET/CT is an emerging diagnostic tool that could soon be the new gold standard for imaging PCa. The production of images with striking tumour to background contrast was exemplified by Prof Michael Hofman, Peter MacCallum Cancer Centre, Melbourne, Australia; during the first 'Game Changing' session he presented the case of

"MRI is an excellent tool to help us in finding the most relevant cancer in the prostate because we not only can look at anatomy, but also cell density and perfusion" 
a male with Grade Group 4 PCa. Through conventional CT imaging, numerous pelvic and abdominal subcentimetre nodes were "barely visible," but when using PSMA PET/ CT they were "lighting up very brightly," highlighted Prof Hofman. In the second presentation of the session, Dr Matthias Heck, Technical University of Munich, Munich, Germany, drew attention to the fact that with CT/MRI, lymph nodes are only treated as suspicious if they are $>8-10 \mathrm{~mm}$ in size. ${ }^{5}$ Given that up to $80 \%$ of pelvic lymph node metastases are $<8 \mathrm{~mm}$, this is a crucial drawback to the use of $\mathrm{CT} / \mathrm{MRI}$ in the PCa diagnostic pathway, meaning that the capability of PSMA PET to detect lymph node metastases of only a few $\mathrm{mm}$ is an important advantage of its use for early detection of severe metastatic disease. ${ }^{6}$ However, the clinical benefit of such early detection is yet to be proven.

\section{proPSMA}

Prof Hofman presented the recent data from the proPSMA trial that analysed the diagnostic validity of PSMA PET/CT in males across Australia with untreated, biopsy-proven PCa. ${ }^{7}$ A 27\% greater accuracy for PSMA PET/CT versus CT plus bone scan (CT+BS), the current standard of care, was reported (92\% versus 65\%, respectively), which was a result of higher sensitivity and specificity.

Of note in the secondary endpoints, management change, for example from surgery to androgen deprivation therapy, occurred in $28 \%$ of males in the PSMA PET/CT arm versus only $15 \%$ in the $\mathrm{CT}+\mathrm{BS}$ arm. Additionally, uncertain findings were reported in $23 \%$ of males in the $C T+B S$ arm, compared with only $7 \%$ in PSMA PET/CT, and a reduced radiation dose was needed for PSMA PET/CT (8 mSv versus $19 \mathrm{mSv}$ ). At second-line crossover imaging, it was shown that the endpoint of management change was maintained in PSMA $\mathrm{PET} / \mathrm{CT}$, and that there was little value in having a $\mathrm{CT}+\mathrm{BS}$ following PSMA PET/CT.

While preliminary data are promising, there is still a lack of prospective or multicentre data. Furthermore, both Prof Hofman and Dr Heck noted that the sensitivity of PSMA PET/CT in the proPSMA trial is speculated to be overestimated because the investigators were unable to define the true incidence of pelvic nodal disease as no males underwent pelvic node dissection. Prof Hofman concluded his session with acknowledgement that the cost effectiveness
Through conventional CT imaging,

numerous pelvic and abdominal

subcentimetre nodes were "barely

visible," but when using PSMA

PET/CT they were "lighting up very brightly"

and availability of PSMA PET/CT needs to be investigated, but commented: "In summary, the proPSMA data supports PSMA PET/CT as a replacement to current standard of care CT+BS."

\section{SUMMARY}

The paradigm shift towards an image-guided $\mathrm{PCa}$ diagnostic pathway is set to drastically change $\mathrm{PCa}$ practice. The constant drive to improve outcomes of PCa and metastatic disease has produced innovative solutions, each with improving diagnostic sensitivity and specificity. While MRI has been hailed as the current gold standard, there is plentiful room for new or reinvented imaging modalities to better MRI and hold this accolade.

\section{References}

1. Ahmed HU et al. Diagnostic accuracy of multi-parametric MRI and TRUS biopsy in prostate cancer (PROMIS): a paired validating confirmatory study. Lancet. 2017;389(10071):815-22.

2. Radtke JP et al. Combined clinical parameters and multiparametric magnetic resonance imaging for advanced risk modeling of prostate cancer-patienttailored risk stratification can reduce unnecessary biopsies. Eur Urol. 2017;72(6):888-96.

3. Mannaerts CK et al. Detection of clinically significant prostate cancer in biopsy-naïve men: direct comparison of systematic biopsy, multiparametric MRI- and contrastultrasound-dispersion imaging-targeted biopsy. BJU Int. 2O2O;DOI:10.1111/bju.15093. [Epub ahead of print].

4. Mannaerts CK et al. Multiparametric ultrasound for prostate cancer detection and localization: correlation of B-mode, shear wave elastography and contrast enhanced ultrasound with radical prostatectomy specimens. J Urol. 2019;202(6):1166-73.

5. Heesakkers RAM et al. MRI with a lymph-nodespecific contrast agent as an alternative to $\mathrm{CT}$ scan and lymph-node dissection in patients with prostate cancer: a prospective multicohort study. Lancet Oncol. 2008;9(9):850-6.

6. Jilg CA et al. Diagnostic accuracy of Ga-68-HBEDCC-PSMA-ligand-PET/CT before salvage lymph node dissection for recurrent prostate cancer. Theranostics. 2017;7(6):1770-80.

7. Hofman MS et al. Prostate-specific membrane antigen PET-CT in patients with high-risk prostate cancer before curative-intent surgery or radiotherapy (proPSMA): a prospective, randomised, multicentre study. Lancet. 2020;395(10231):1208-16. 